\title{
User Selection for NOMA based MIMO with Physical Layer Network Coding in Internet of Things Applications
}

\author{
Saadet Simay Yılmaz, Berna Özbek*, Mert İlgüy, Bismark Okyere, Leila Musavian, Jonathan Gonzalez
}

\begin{abstract}
Non-orthogonal multiple access (NOMA) based multiple-input multiple-output (MIMO), which has the potential to provide both massive connectivity and high spectrum efficiency, is considered as one of the efficient techniques for sixth generation (6G) wireless systems. In massive Internet of Things (IoT) networks, user-set selection is crucial for enhancing the overall performance of NOMA based systems when compared with orthogonal multiple access (OMA) techniques. In this paper, we propose a user-set selection algorithm for IoT uplink transmission to improve the sum data rate of the NOMA based MIMO systems. In order to exchange data between the selected IoT pairs, we propose to employ wireless physical layer network coding (PNC) to further improve the spectral efficiency and reduce the delay to fulfill the requirements of future IoT applications. Performance evaluations are provided based on both sum data rate and bit error rate for the proposed NOMA based MIMO with PNC in the considered massive IoT scenarios.
\end{abstract}

Index Terms-User-Set Selection, MIMO, NOMA, PNC, IoT.

\section{INTRODUCTION}

I NTERNET of Things (IoT), including sensors, radio frequency identification (RFID), actuators and industrial equipment, exchange information through the integration of Internet and wireless networks while establishing connections between things and between people and things [1]. Sixth generation $(6 \mathrm{G})$ networks are visioned to provide high-quality transmission for massive number of wireless nodes via IoT devices, taking into account the constraints of reliability [2] and security [3] [4] through the usage of heterogeneous computing systems to balance energy power consumption and efficiency [5].

Non-orthogonal multiple access (NOMA) is investigated as one of the possible enabling multiple access technologies to fulfill the $6 \mathrm{G}$ requirements such as high data rate, ultra-low latency, large connectivity while enhancing overall spectral efficiency. The main concept of NOMA is to simultaneously serve multiple users through the same time, same frequency or same code [6]. In literature, there are mainly two different types of NOMA technologies: Power-domain NOMA [7] and code-domain NOMA [8]. In this work, we consider power

Copyright (c) 2021 IEEE. Personal use of this material is permitted. However, permission to use this material for any other purposes must be obtained from the IEEE by sending a request to pubs-permissions@ieee.org. *Corresponding author: B.Özbek.

S. S. Yilmaz, B. Özbek, M. İlgüy are with the Dept. of Electrical and Electronics Eng., Izmir Institute of Technology, Turkey

B. Okyere and L. Musavian are with the School of Computer Science and Electronic Engineering (CSEE), University of Essex, UK.

J. Gonzalez is with GS-Lda, 3750-101, Àgueda, Portugal. domain NOMA in which the power difference of the users is exploited for multi-user multiplexing while applying successive interference cancellation (SIC) to mitigate the inter-user interference [9].

Since NOMA employs more than one user at the same resource block, it can improve the sum data rate performance compared to its traditional counterpart, orthogonal multiple access (OMA) [7] [10]-[12]. Besides, the latency performance of power-domain NOMA versus OMA has been also examined in recent literature. Specifically, the work presented in [13] investigated the link-layer capacity, namely, the effective capacity, of NOMA under probabilistic delay constraints and showed that NOMA outperforms OMA at high signal to noise ratios. Further investigations have considered the NOMA networks in finite blocklength packet regimes and investigated the performance of NOMA networks in terms of ultra reliable low latency communications requirements [14].

Despite the noticeable advantages of NOMA, other advanced technologies are also needed to achieve higher spectral efficiency. One of these technologies is multiple input multiple output (MIMO) where multiple antennas at the base station (BS) can be used to simultaneously serve many users while separating them in the spatial domain. Since MIMO provides extra degrees of freedom, the integration of NOMA and MIMO has recently become an attractive solution in terms of massive connectivity support. In the combined MIMO-NOMA scheme, users are divided into clusters which include both near and far users. For downlink transmission, linear precoding schemes can be employed at the BS to suppress inter-cluster interference while SIC-based detectors are applied at the user's side to mitigate the intra-cluster users' interference [15]. For uplink transmission, on the other hand, both SIC-based detectors and postcoding techniques are performed at the BS.

For NOMA-based MIMO networks, adaptive user selection and power allocation are critical to improve the performance of overall networks. In order to allocate the users to their clusters, the sorting algorithm was presented in [16] and the combination of the semi-orthogonal user selection (SUS) method with user matching technique was examined in [17]. The performance of SUS algorithm with specified regions for the weak and strong users was investigated for downlink MIMO-NOMA in [18].

Physical layer network coding (PNC) is one of the promising techniques to ensure network reliability and reduce the delay for 6G wireless systems [19] [20]. PNC has been investigated mostly in two-way relay channels. Two-way relay 
channels consist of one relay node and two terminal nodes which are subject to communicate via a relay node. Power consumption can be reduced by dividing the total distance between the terminal nodes into smaller distances to improve the overall system capacity. The exchange of the data between the terminal nodes is hold through two transmission phases. The transmission from terminal nodes to the relay node is named multiple access channel (MAC) phase while the transmission from the relay node to the terminal nodes is called broadcast channel (BC) phase. In this paper, the relay node and the terminal nodes are the BS and IoT devices, respectively. In addition to that, one of the IoT devices can act as a relay to share data between an IoT pair.

The data exchange in two-way relay channels were studied mainly with three different transmission schemes. These schemes are the conventional four time slot transmission, network coding (NC) and PNC. In these three types of transmissions, PNC is the most spectral efficient scheme since all devices send their data simultaneously to the BS. Then, the whole transmission is completed in two time slots. For MAC phase, which occurs in the first time slot, users transmit their data to the BS. Then, the BS makes a decision on the network coded symbol (NCS) based on the combined constellation which will be used to extract the other user's data in the second time slot. For the BC phase, which occurs in the second time slot, the BS broadcasts NCS to all devices. Then, all devices estimate their pairs' data by performing network coding operation. In [21], spectral efficiency of PNC was examined while both bit error rate (BER) and sum data rate of PNC were investigated in [22]. Since the decision of the NCS is done based on the received constellation, the performance of PNC depends on the modulation scheme. In [23]-[25], BER performances of quadrature phase shift keying (QPSK), M-ary phase shift keying (M-PSK) and Mary quadrature amplitude modulation (M-QAM) based PNC were examined using closed-form expressions. Asynchronous PNC was studied in [26] as part of an IoT architecture. In this work, we consider PNC systems under perfect synchronisation for massive IoT applications.

In [27], PNC and multiple antenna technology were combined to boost the network performance. The NCS is detected through zero forcing (ZF) and minimum mean squared error (MMSE) based linear equalization techniques. Specifically, the equalization matrices of ZF and MMSE consist of sumdifference matrix to obtain sum and difference data of the user pairs at the BS. Thereby, the log likelihood ratio (LLR) is obtained by using the sum and difference signals. Then, the BS decides on NCS based on the sign of the LLR. In [28], multiple antenna system were extended to multiuser massive MIMO for PNC systems. It was demonstrated that PNC outperforms NC considering BER performances of both multi-user MIMO and multiuser massive MIMO. In [29], the performance of PNC combined with NOMA was investigated for single antenna systems. The combination of NOMA and PNC with cascade-computation decoding scheme without employing multiple antennas was studied in [30].

In this paper, we propose to combine the NOMA-MIMO systems with PNC to exchange information among several
IoT devices efficiently. We focus on uplink transmission and propose a novel user-set selection in massive IoT environment for $6 \mathrm{G}$ applications. The contributions of this work are summarized in the following:

- NOMA based MIMO systems with PNC is proposed and its performance is investigated. To the best of the authors' knowledge, this is the first work the implement PNC in NOMA MIMO networks.

- We then propose a novel user-set selection algorithm for NOMA-based MIMO systems in uplink transmission and demonstrate the sum data rate results in massive IoT applications.

- We combine the proposed user-set selection algorithm with PNC in IoT applications and provide BER results for the proposed pairing scenario.

The remainder of this paper is organized as follows. Uplink NOMA-based MIMO system model is detailed in Section II. Then, we propose the user-set selection and power allocation scheme for uplink NOMA based MIMO system in Section III. Next, the proposed NOMA based MIMO-PNC is discussed in Section IV. Simulation results and discussion are provided in Section V. Finally, concluding remarks are drawn in Section VI.

\section{SySTEM MODEL}

We consider an uplink MIMO-NOMA communication in which a BS has $\mathrm{N}$ antennas and is serving multiple IoT devices as shown in Fig. 1. There are $K$ IoT devices having a singleantenna associated with $K \geq 2 N$.

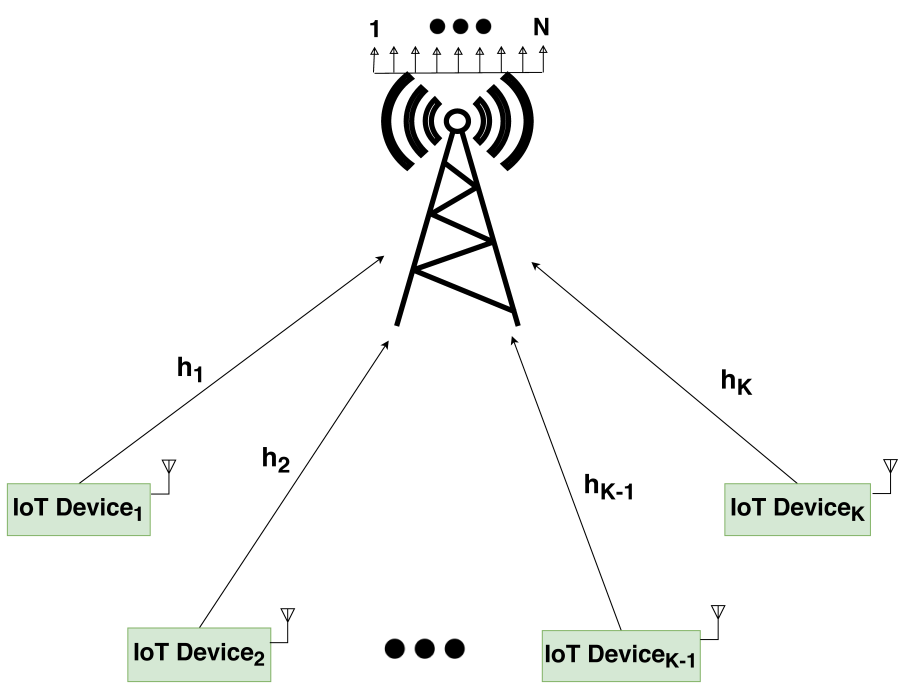

Fig. 1: Uplink NOMA based MIMO System Model.

The BS with $\mathrm{N}$ antennas can serve up to $\mathrm{N}$ IoT devices with traditional OMA systems. On the other hand, in the uplink NOMA system, the BS simultaneously supports two user sets. As a result, the total number of IoT devices is equal to $2 \mathrm{~N}$ [31]. Thus, the selection of $\mathrm{N}$ IoT devices for each set among $\mathrm{K}$ total IoT devices is critical and is proposed in Section III.

There are two user sets as the strong and weak where each of them includes $\mathrm{N}$ IoT devices. The strong user set represents 
the IoT devices that have high channel gains, whereas the IoT devices in the weak user set have relatively low channel gains. The channel matrices of strong and weak user sets are denoted as $\mathbf{H}_{1}$ and $\mathbf{H}_{2}$, respectively.

$$
\mathbf{H}_{1}=\left[\mathbf{h}_{1,1} \ldots \mathbf{h}_{n, 1} \ldots \mathbf{h}_{N, 1},\right]
$$

and

$$
\mathbf{H}_{2}=\left[\mathbf{h}_{1,2} \ldots \mathbf{h}_{m, 2} \ldots \mathbf{h}_{N, 2}\right],
$$

where $\mathbf{h}_{n, j}=L_{n, j} \mathbf{g}_{n, j}$ is the uplink channel vector of size $N \times 1$ between BS and an IoT device out of $n=1,2, \ldots, N$ in the set of $j=1,2$. For IoT device $n$ in the set $j$, the path-loss coefficient is defined by $L_{n, j}$ and $\mathbf{g}_{n, j}$ represents the channel coefficient which is modelled by complex Gaussian distribution as $\mathcal{C N}(0,1)$.

The received signal at the $\mathrm{BS}$ is the summation of the signals coming from these two sets as:

$$
\mathbf{y}=\left(\mathbf{H}_{1} \mathbf{s}_{1}+\mathbf{H}_{2} \mathbf{s}_{2}\right)+\mathbf{n},
$$

where $\mathbf{s}_{1}=\left[\sqrt{\alpha_{1}} s_{1,1} \cdots \sqrt{\alpha_{1}} s_{N, 1}\right]^{T}$ represents the transmitted signal vectors of size $N \times 1$ for the strong user set and $\mathbf{s}_{2}=\left[\sqrt{\alpha_{2}} s_{1,2} \cdots \sqrt{\alpha_{2}} s_{N, 2}\right]^{T}$ denotes the transmitted signal vectors of size $N \times 1$ for the weak user set. The uplink symbol of IoT device $n$ in the corresponding set $j$ is given by $s_{n, j}$ with $\mathbb{E}\left[\left|s_{n, j}\right|^{2}\right] \leq P$ where $P$ is the maximum transmit power per IoT device. Power allocation factors for the strong and weak user sets are defined by $\alpha_{1}$ and $\alpha_{2}$ with $0<\alpha_{1}, \alpha_{2} \leq 1$, respectively. The additive white Gaussian noise (AWGN) vector of size $N \times 1$ is given by $\mathbf{n}$ and each element of the vector is modelled by a Gaussian distribution random variable with zero mean and $\sigma_{n}^{2}$ variance.

According to the received signal in (3), both symbols belonging to the strong and weak users are extracted at the BS. Firstly, the signals of the IoT devices in the strong user set are decoded under the inter-set interference caused by the devices in the weak user set. Then, the SIC is applied by using the strong users' signals to decode the signals in the weak user set. Thus, the signals in the weak user set are decoded without the inter-set interference.

In order to cancel the inter-user interference between strong and weak users, ZF postcoding is employed at the BS. Assuming perfect channel state information (CSI) belonging to all IoT devices is available, $\mathrm{ZF}$ postcoding matrix $\mathbf{W}_{j}^{\prime}$ with $j=1,2$ is determined through overall channel matrix by,

$$
\mathbf{W}_{j}^{\prime}=\mathbf{H}_{j}^{H}\left(\mathbf{H}_{j} \mathbf{H}_{j}^{H}\right)^{-1},
$$

where the $\mathrm{ZF}$ postcoding matrix is given by

$$
\mathbf{W}_{j}=\left[\mathbf{w}_{1, j}{ }^{T} \mathbf{w}_{2, j}{ }^{T} \ldots \mathbf{w}_{N, j}{ }^{T}\right]^{T},
$$

with $\mathbf{w}_{n, j}$ being the $\mathrm{ZF}$ postcoder with the size of $1 \times N$ for the IoT device $n$ in the corresponding set $j$. It is given by $\mathbf{w}_{n, j}=\frac{\mathbf{w}_{n, j}^{\prime}}{\left\|\mathbf{w}_{n, j}^{\prime}\right\|}$ where $\mathbf{w}_{n, j}^{\prime}$ is the nth column of $\mathbf{W}_{j}^{\prime}$.

Firstly, the signals of the strong IoT devices are decoded under inter-set interference by using the postcoding matrix $\mathbf{W}_{1}$. Then, the received signal vector of $\mathbf{r}_{1}=\left[\begin{array}{ll}r_{1,1} & r_{2,1} \ldots r_{N, 1}\end{array}\right]^{T}$ is obtained by

$$
\mathbf{r}_{1}=\mathbf{W}_{1} \mathbf{y}=\mathbf{W}_{1} \mathbf{H}_{1} \mathbf{s}_{1}+\mathbf{W}_{1} \mathbf{H}_{2} \mathbf{s}_{2}+\mathbf{W}_{1} \mathbf{n} .
$$

From (6), in the strong user set, the received signal of the device $n$ is given by:

$r_{n, 1}=\mathbf{w}_{n, 1} \mathbf{h}_{n, 1} \sqrt{\alpha_{1}} s_{n, 1}+\left(\sum_{i=1}^{N} \mathbf{w}_{n, 1} \mathbf{h}_{i, 2} \sqrt{\alpha_{2}} s_{i, 2}\right)+\mathbf{w}_{n, 1} \mathbf{n}$,

where the first term represents the desired signals of the strong user set and the second term is the inter-set interference caused by the weak user set.

The received instantaneous signal to interference plus noise ratio (SINR) for device $n$ in the strong user set is given by

$$
\operatorname{SINR}_{n, 1}=\frac{\left|\mathbf{w}_{n, 1} \mathbf{h}_{n, 1}\right|^{2} \alpha_{1} P}{\alpha_{2} P \sum_{i=1}^{N}\left|\mathbf{w}_{n, 1} \mathbf{h}_{i, 2}\right|^{2}+\alpha_{1} P \sum_{\substack{j=1, j \neq n}}^{N}\left|\mathbf{w}_{n, 1} \mathbf{h}_{j, 1}\right|^{2}+\sigma_{n}^{2}} .
$$

For decoding of the signals of the weak user set, SIC is applied and, in this case, there will be no inter-set interference. Thus, after utilizing $\mathrm{ZF}$ postcoding matrix $\mathbf{W}_{2}$, the received signal vector of $\mathbf{r}_{2}=\left[\begin{array}{lll}r_{1,2} & r_{2,2} \ldots r_{N, 2}\end{array}\right]^{T}$ is given by

$$
\mathbf{r}_{2}=\mathbf{W}_{2} \mathbf{H}_{2} \mathbf{s}_{2}+\mathbf{W}_{2} \mathbf{n} \text {. }
$$

From (9), for the weak user set, the received signal of the device $n$ is given by

$$
r_{n, 2}=\mathbf{w}_{n, 2} \mathbf{h}_{n, 2} \sqrt{\alpha_{2}} s_{n, 2}+\mathbf{w}_{n, 2} \mathbf{n} .
$$

The received instantaneous SINR for the IoT device $n$ in the weak user set is expressed by:

$$
\operatorname{SINR}_{n, 2}=\frac{\left|\mathbf{w}_{n, 2} \mathbf{h}_{n, 2}\right|^{2} \alpha_{2} P}{\alpha_{2} P \sum_{\substack{j=1, j \neq n}}^{N}\left|\mathbf{w}_{n, 2} \mathbf{h}_{j, 2}\right|^{2}+\sigma_{n}^{2}} .
$$

The sum data rate of $N$ IoT devices for each user set, $j$, is hence given by,

$$
R_{j, \mathrm{NOMA}}=\sum_{n=1}^{N} \log _{2}\left(1+\operatorname{SINR}_{n, j}\right) .
$$

Thus, the overall sum data rate is determined by

$$
R_{\mathrm{sum}}^{\mathrm{NOMA}}=\sum_{j=1}^{2} R_{j, \mathrm{NOMA}} .
$$

For the purpose of comparing, the data rates of IoT devices are given in the corresponding sets for conventional OMA system as,

$$
R_{j, \mathrm{OMA}}=\frac{1}{2} \sum_{n=1}^{N} \log _{2}\left(1+\frac{\left|\mathbf{w}_{n, j} \mathbf{h}_{n, j}\right|^{2} P}{\sigma_{n}^{2}}\right),
$$

where $\frac{1}{2}$ is added since two time slots are required to support $2 N$ devices in the OMA system with $N$ antennas, whereas NOMA can support $2 N$ devices during a single time slot. Therefore, the overall sum data rate for OMA is given by,

$$
R_{\mathrm{sum}}^{\mathrm{OMA}}=\sum_{j=1}^{2} R_{j, \mathrm{OMA}}
$$




\section{Proposed User-Set Selection For NOMA-BASEd MIMO SYSTEMS}

In this section, we propose a user-set selection algorithm to improve the performance of the uplink MIMO-NOMA system.

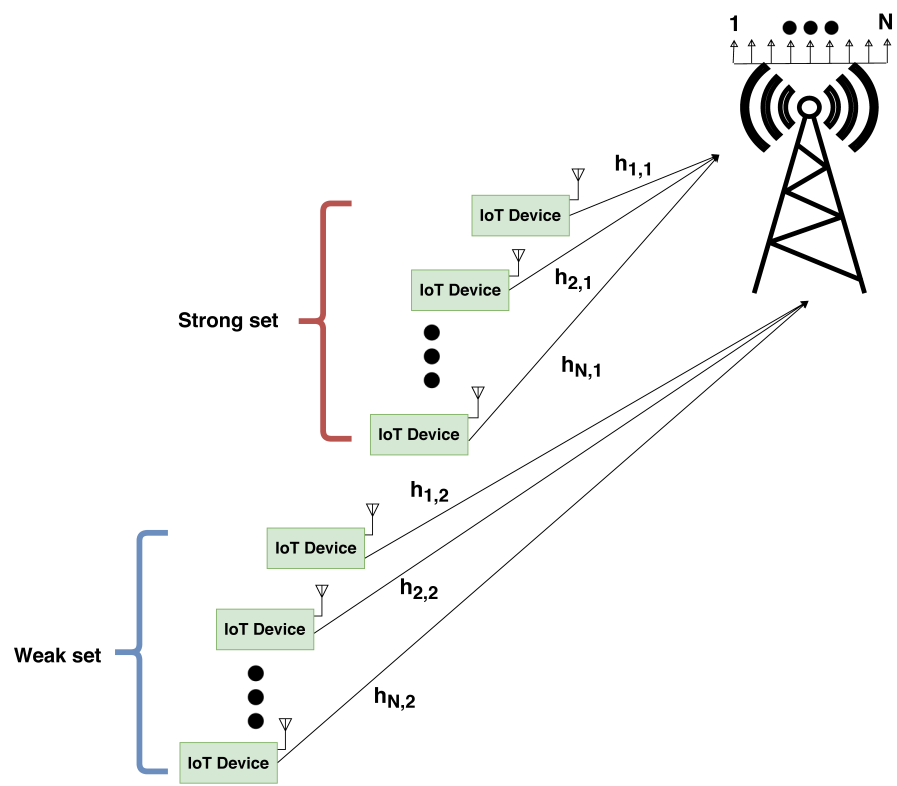

Fig. 2: Proposed user set selection for Uplink NOMA based MIMO.

As illustrated in Fig. 2, the proposed user-set selection algorithm selects totally $2 N$ IoT devices without affecting the transmission of strong users. We firstly determine the strong and weak user sets considering their channel gains. Then, we select $N$ IoT devices from each set based on both the channel gain and the correlation properties to mitigate inter-set interference. After that, we determine the optimal power values $\alpha_{1}$ and $\alpha_{2}$ for both strong and weak user sets, respectively.

\section{A. Proposed User-Set Selection Method}

A total of $N$ IoT devices having higher channel gains in the strong user set are selected. IoT devices in the weak user set cause inter-set interference to Io $\mathrm{T}$ devices in the strong user set. Therefore, the proposed user-set selection algorithm determines weak users through an elimination procedure based on the channel correlation between strong and weak user sets. Orthogonal IoT devices in these two sets are selected so that inter-set interference can be reduced. In order to select $N$ IoT devices for weak user set, the BS compares their orthogonality with the selected IoT devices in strong user set.

Details of the proposed method are explained below:

- Step 1: Channel gains of $K$ IoT devices are sorted in descending order:

$$
\mathcal{S}_{\text {ord }}=\left\{\left\|\mathbf{h}_{k}\right\| \mid\left\|\mathbf{h}_{k}\right\|>\left\|\mathbf{h}_{k+1}\right\| ; k \in\{1,2, \ldots, K\}\right\} .
$$

- Step 2: Utilizing $\mathcal{S}_{\text {ord }}$, BS classifies the $K$ devices into two groups. The set of devices with the higher channel gains is represented by $\mathcal{S}_{\mathrm{s}}$, whereas $\mathcal{S}_{\mathrm{w}}$ includes the set of devices with the lower channel gains.

$$
\mathcal{S}_{s}=\left\{\left\|\mathbf{h}_{1}\right\|, \ldots,\left\|\mathbf{h}_{\lfloor K / 2\rfloor}\right\|\right\}
$$

in here $\lfloor$.$\rfloor indicates the floor function.$

$$
\mathcal{S}_{w}=\left\{\left\|\mathbf{h}_{\lfloor K / 2\rfloor+1}\right\|, \ldots,\left\|\mathbf{h}_{K}\right\|\right\} .
$$

- Step 3: From $\mathcal{S}_{\mathrm{s}}$, strong user set is formed by selecting the first $N$ devices,

$$
\mathbf{H}_{1}=\left\{\mathbf{h}_{n} \mid \mathbf{h}_{n} \in \mathcal{S}_{s}, n=1, \ldots, N\right\} .
$$

- Step 4: BS determines the degrees of orthogonality $\beta_{n, i}$ between IoT device $n$ in strong user set and IoT device $i$ in set of $\mathcal{S}_{\mathrm{w}}$ :

$$
\beta_{n, i}=\frac{\left|\mathbf{h}_{n}^{H} \mathbf{h}_{i}\right|}{\left\|\mathbf{h}_{n}\right\|\left\|\mathbf{h}_{i}\right\|}
$$

where $n=1, \ldots, N$ and $i=\lfloor K / 2\rfloor+1, \ldots, K$. When $\beta_{n, i}$ is equal to zero, two channel vectors are orthogonal to each other. When this value is closer to 1 , two channel vectors are in similar directions and then they are highly correlated.

- Step 5: As in [32], a set of $\mathcal{U S}_{i}$ that satisfy the following criterion for $i^{\text {th }}$ device in $\mathcal{S}_{w}$ is constructed:

$$
\mathcal{U S}_{i}=\left\{i \in \mathcal{S}_{w} \mid \beta_{n, i}>\beta ; \forall n\right\}
$$

where $\beta$ is a fixed threshold holding a value between 0 and 1 . As a result, $\mathcal{U} \mathcal{S}_{i}$ includes $i^{\text {th }}$ weak device that is correlated to the selected strong users.

- Step 6: For the $i^{t h}$ device, if the set of $\mathcal{U} \mathcal{S}_{i}$ is not empty, $i^{\text {th }}$ IoT device is removed from the set of $\mathcal{S}_{\mathrm{w}}$ by,

$$
\mathcal{S}_{w}=\mathcal{S}_{w} \backslash\left\{\mathbf{h}_{i}\right\} \text {, if Card }\left(\mathcal{U S}_{i}\right) \neq 0
$$

Then, $\mathcal{S}_{w}$ includes the set of weak devices that are orthogonal to the selected strong users.

- Step 7a: If the cardinality of $\mathcal{S}_{w}$ is equal or higher than $N$, the proposed user set selection algorithm selects the IoT devices in the weak user set as follows:

$$
\mathbf{H}_{2}=\left\{\mathbf{h}_{k} \mid \mathbf{h}_{k} \in \mathcal{S}_{w}, k=1, \ldots, N\right\}
$$

Then, the NOMA-based MIMO transmission is performed for the selected IoT devices in (19) and (23).

- Step 7b: If the cardinality of $\mathcal{S}_{w}$ is less than $N$, the IoT devices in the weak user set are selected from the set of $\mathcal{S}_{\text {ord }}$ by,

$\mathbf{H}_{2}=\left\{\mathbf{h}_{m} \mid \mathbf{h}_{m} \in \mathcal{S}_{\text {ord }}, m=\left\lfloor\frac{K}{2}\right\rfloor+1, \ldots,\left\lfloor\frac{K}{2}\right\rfloor+N\right\}$.

Then, the OMA-based MIMO transmission is performed for the selected IoT devices in (19) and (24). 


\section{B. Power Allocation Scheme}

In this section, we examine an optimal power allocation to find the coefficients of $\alpha_{1}$ and $\alpha_{2}$. Our objective function is to maximize the sum data rate of IoT devices in NOMA based MIMO system, defined by

$$
f(\boldsymbol{\alpha})=R_{1, \mathrm{NOMA}}+R_{2, \mathrm{NOMA}}
$$

where $\boldsymbol{\alpha}=\left[\alpha_{1}, \alpha_{2}\right]$.

The sum data rate maximization problem subject to the data rate constraint based on OMA-based MIMO system is given by

$$
\begin{array}{ll} 
& \max _{\boldsymbol{\alpha}} f(\boldsymbol{\alpha}) \\
\text { s.t. } & R_{1, \mathrm{NOMA}} \geq R_{1, \mathrm{OMA}}, \\
& R_{2, \mathrm{NOMA}} \geq R_{2, \mathrm{OMA}}, \\
& 0<\alpha_{1}, \alpha_{2} \leq 1 .
\end{array}
$$

Constraints (27a) and (27b) give the minimal data rate constraints in which the achievable data rate in uplink NOMA should be no less than a conventional OMA system. Constraint (27c) gives the interval of the $\alpha_{1}$ and $\alpha_{2}$. The maximization function in (26) is equivalent to minimizing $-\mathrm{f}($.$) , thus the$ corresponding optimization problem can be solved efficiently with the standard nonlinear programming optimization tools [33]. The minimum of a constrained nonlinear multivariate function can be found using the interior-point method. As a result of the interior-point method, the optimum output values are obtained as $\alpha_{1}^{*}$ and $\alpha_{2}^{*}$ to provide the maximum sum data rate under the given constraints.

\section{Proposed NOMA BASEd MIMO-PNC}

In this section, we present the system model for the proposed NOMA based MIMO-PNC. Through the proposed user set selection, two sets including $N$ strong and $N$ weak IoT devices are constructed. The paired IoT devices communicate via BS. Here, we consider pairing scenario called SS-WW where each strong IoT device is paired with another strong IoT device and each weak IoT device is paired with another weak IoT device. In Fig. 3, SS-WW pairing is illustrated for the case of $N=2$ and $K=4$.

In uplink transmission, the NCS vector of $\mathbf{s}_{R} \in \mathbb{C}^{Q \times 1}$ whose dimension is equal to $Q=N$ is estimated at BS. It is assumed that BS has perfect CSI, IoT devices transmit their signals simultaneously to BS with perfect synchronization, and all IoT devices are modulated by QPSK.

We define the composite transmit symbol vector of $\mathbf{s} \in$ $\mathbb{C}^{2 Q \times 1}$, which is obtained through concatenation of transmit symbol vector of the strong user set $\mathbf{s}_{1}$ and the weak set $\mathbf{s}_{2}$ :

$$
\mathbf{s}=\left[\begin{array}{l}
\mathbf{s}_{1} \\
\mathbf{s}_{2}
\end{array}\right]=\left[\begin{array}{c}
\mathbf{s}_{1,1} \\
\mathbf{s}_{1,2} \\
\ldots \\
\mathbf{s}_{1, Q} \\
\mathbf{s}_{2,1} \\
\mathbf{s}_{2,2} \\
\ldots \\
\mathbf{s}_{2, Q}
\end{array}\right] .
$$
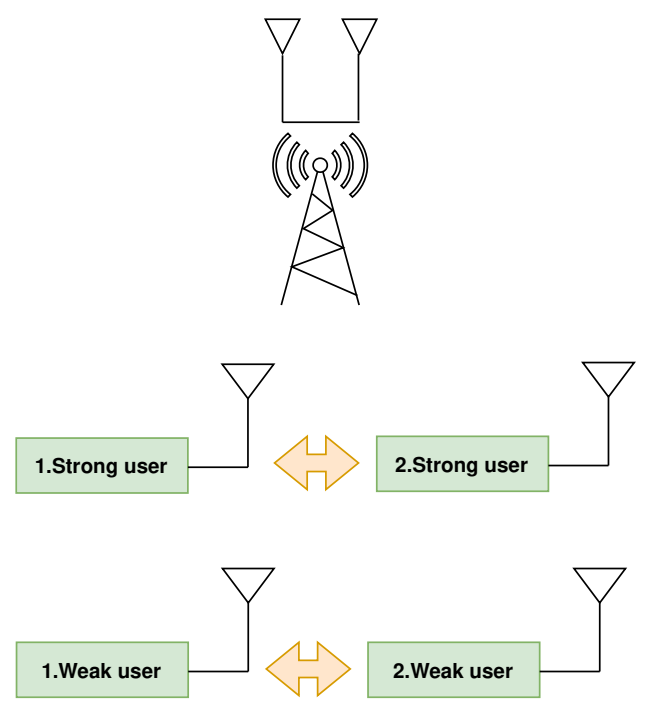

Fig. 3: Pairing Scenario for $N=2$ and $K=4$

For SS-WW pairing scenario, the NCS vector is given by

$$
\mathbf{s}_{R}=\left[\begin{array}{c}
\mathbf{s}_{1,1} \oplus \mathbf{s}_{1,2} \\
\mathbf{s}_{1,3} \oplus \mathbf{s}_{1,4} \\
\ldots \\
\mathbf{s}_{1, Q-1} \oplus \mathbf{s}_{1, Q} \\
\mathbf{s}_{2,1} \oplus \mathbf{s}_{2,2} \\
\mathbf{s}_{2,3} \oplus \mathbf{s}_{2,4} \\
\ldots \\
\mathbf{s}_{2, Q-1} \oplus \mathbf{s}_{2, Q}
\end{array}\right] .
$$

The received vector $\mathbf{y} \in \mathbb{C}^{N \times 1}$ in (3) is rewritten as follows:

$$
\mathbf{y}=\mathbf{C s}+\mathbf{n},
$$

where $\mathbf{C}$ is the composite channel matrix with a dimension of $N \times 2 Q$. Then, the received vector can be arranged as:

$$
\begin{aligned}
\mathbf{y} & =\left(\mathbf{C D}^{-1}\right)(\mathbf{D s})+\mathbf{n} \\
& =\hat{\mathbf{C}} \hat{\mathbf{s}}+\mathbf{n},
\end{aligned}
$$

where $\mathbf{D} \in \mathbb{C}^{2 Q \times 2 Q}$ is the sum difference matrix, $\hat{\mathbf{C}} \in$ $\mathbb{C}^{N \times 2 Q}$ is manipulated composite channel matrix and $\hat{\mathbf{s}} \in$ $\mathbb{C}^{2 Q \times 1}$ is the target estimated vector.

We propose to construct the sum-difference matrix according to

$$
\mathbf{D}=\left[\begin{array}{ccc}
\mathbf{D}_{2 \times 2} & 0 & 0 \\
0 & \ddots & 0 \\
0 & 0 & \mathbf{D}_{2 \times 2}
\end{array}\right]
$$

where $\mathbf{D}_{2 \times 2}$ is given by

$$
\mathbf{D}_{2 \times 2}=\left[\begin{array}{cc}
1 & 1 \\
1 & -1
\end{array}\right] .
$$

Then, the manipulated composite channel matrix is found as

$$
\hat{\mathbf{C}}=\left[\begin{array}{ll}
\hat{\mathbf{H}}_{1} & \hat{\mathbf{H}}_{2}
\end{array}\right] .
$$


Besides, the target estimated vector is found according to

$$
\hat{\mathbf{s}}=\left[\begin{array}{c}
\mathbf{s}_{1,1}+\mathbf{s}_{1,2} \\
\mathbf{s}_{1,1}-\mathbf{s}_{1,2} \\
\cdots \\
\mathbf{s}_{1, Q-1}+\mathbf{s}_{1, Q} \\
\mathbf{s}_{1, Q-1}-\mathbf{s}_{1, Q} \\
\mathbf{s}_{2,1}+\mathbf{s}_{2,2} \\
\mathbf{s}_{2,1}-\mathbf{s}_{2,2} \\
\cdots \\
\mathbf{s}_{2, Q-1}+\mathbf{s}_{2, Q} \\
\mathbf{s}_{2, Q-1}-\mathbf{s}_{2, Q}
\end{array}\right] .
$$

After that, we perform either ZF or MMSE based equalization to detect the NCS symbols belonging to the strong and weak user pairs separately. For ZF and MMSE, the equalization matrix of $\hat{\mathbf{W}}_{i} ; \forall i \in\{1,2\}$ are given respectively:

$$
\begin{gathered}
\hat{\mathbf{W}}_{i}=\left(\hat{\mathbf{H}}_{i}^{H} \hat{\mathbf{H}}_{i}\right)^{-1} \hat{\mathbf{H}}_{i}^{H} . \\
\hat{\mathbf{W}}_{i}=\left(\hat{\mathbf{H}}_{i}^{H} \hat{\mathbf{H}}_{i}+\mathbf{I}_{Q \times Q} \sigma_{n}^{2}\right)^{-1} \hat{\mathbf{H}}_{i}^{H} .
\end{gathered}
$$

Therefore, the equalized vector for the strong user set $\mathbf{r}_{1}$ is calculated as:

$$
\mathbf{r}_{1}=\hat{\mathbf{W}}_{1} \hat{\mathbf{H}}_{1} \hat{\mathbf{s}}_{1}+\hat{\mathbf{W}}_{1} \hat{\mathbf{H}}_{2} \hat{\mathbf{s}}_{2}+\hat{\mathbf{W}}_{1} \mathbf{n}
$$

Also, the equalized vector for the weak set $\mathbf{r}_{2}$ is determined as:

$$
\mathbf{r}_{2}=\hat{\mathbf{W}}_{2} \hat{\mathbf{H}}_{2} \hat{\mathbf{s}}_{2}+\hat{\mathbf{W}}_{2} \mathbf{n}
$$

These two equalized vectors are merged to provide

$$
\mathbf{r}=\left[\mathbf{r}_{1}, \mathbf{r}_{2}\right]^{T}
$$

Then, LLR value of each symbol pair is defined as follows:

$$
\mathrm{LLR}_{q}=\log \left(\mathrm{e}^{\left(\frac{2}{\sigma_{m}^{2}}-\frac{2}{\sigma_{m+1}^{2}}\right)}\left(\frac{\cosh \left(\frac{2 r_{m+1}}{\sigma_{m+1}^{2}}\right)}{\cosh \left(\frac{2 r_{m}}{\sigma_{m}^{2}}\right)}\right)\right),
$$

where $r_{m}, r_{m+1}$ are the elements of the equalized vector $\mathbf{r}$, given in (41), $\forall m \in\{1,3, \cdots, 2 Q-1\}$ and $q=\frac{(m+1)}{2}$ while $\sigma_{m}^{2}$ and $\sigma_{m+1}^{2}$ are the noise variances after the equalization which are given by:

$$
\sigma_{\ell}^{2}= \begin{cases}\left\{\hat{\mathbf{W}}_{1} \hat{\mathbf{W}}_{1}^{H}\right\}_{\ell, \ell} \sigma_{n}^{2}, & \forall \ell \in\{1,2, \cdots Q\} \\ \left\{\hat{\mathbf{W}}_{2} \hat{\mathbf{W}}_{2}^{H}\right\}_{\ell-Q, \ell-Q} \sigma_{n}^{2}, & \forall \ell \in\{Q+1, \cdots 2 Q\}\end{cases}
$$

Finally, based on the LLR value, BS decides on the NCS value of transmitted symbols and forms NCS vector according to the following rule:

$$
\mathbf{s}_{R_{q}}=\left\{\begin{array}{ll}
1, & \operatorname{LLR}_{q} \geq 0 \\
-1, & \text { otherwise }
\end{array}, \quad \forall q \in\{1,2, \cdots, Q\}\right.
$$

\section{Performance Results}

This section presents the simulation results to evaluate the performances of NOMA-based MIMO-PNC systems. The IoT devices are uniformly placed in the cell area which is between $30 \mathrm{~m}$ and $250 \mathrm{~m}$ from the BS. The noise power spectrum density is $-159 \mathrm{dBm} / \mathrm{Hz}$, and the maximum transmit power and the bandwidth of each IoT device are set to $11 \mathrm{dBm}$ and $200 \mathrm{kHz}$ respectively [34]. The path loss is determined by $L=128.1+37.6 \log _{10}\left(d_{n, j}(k m)\right)$ [35] where $d_{n, j}$ is the distance between IoT device $n$ in the set $j$ and the BS. In the OMA-based multiuser MIMO, the strong IoT devices are selected as in (19) while the weak IoT devices are selected as either in (23) or (24). After that, these selected IoT devices establish transmission through two time slots whereas only one time slot is allocated for the NOMA-based multiuser MIMO.

We compare the performance of the proposed user set selection algorithm for multiuser MIMO systems based on NOMA and OMA. Then, the data rate of the strong users is given for the proposed user set selection and the sorting based user set selection for NOMA based systems. The effect of the number of IoT devices, $K$, and the effect of the threshold, $\beta$, on the sum data rate and on the data rate of the strong users are provided.

In Fig. 4, the effect of orthogonality threshold is investigated for $N=2$ and $N=4$. The data rate of the strong users for different threshold values is examined since threshold value directly affects the performance e of the strong user set. It is observed that $\beta=0.7$ provides the highest data rate of the strong users for the case $N=2$ and the weak devices are selected as much as orthogonal to the strong devices. Then, the value of $\beta$ is increased further, the data rate of the strong users decreases due to the increasing inter-set interference with the selection of nearly non-orthogonal devices as weak user set. On the other hand, when $N=4$, the choice of $\beta=0.6$ gives the highest data rate for the strong users due to decreasing the degrees of orthogonality when the number of antennas is increased.

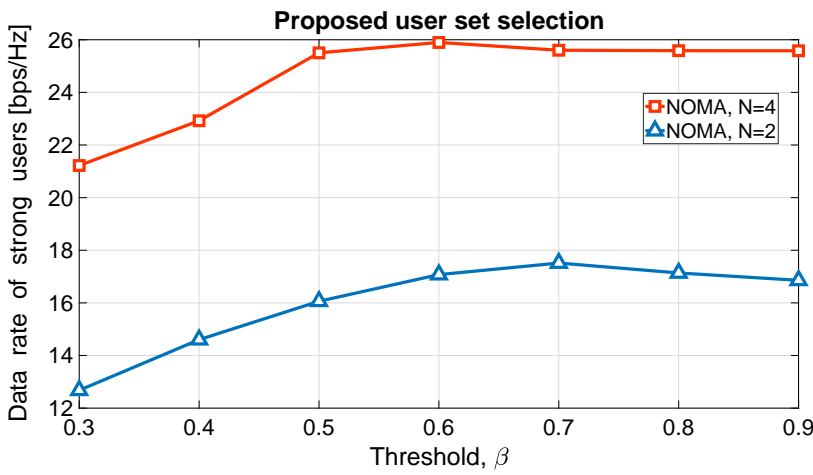

Fig. 4: The data rate of strong users versus the threshold $\beta$, for $K=100$ and different $N$.

Fig. 5 shows the sum data rate of the proposed user set selection through NOMA and OMA for both $N=2$ with $\beta=0.7$ and $N=4$ with $\beta=0.6$ in the densely deployed IoT devices. For the different number of IoT devices and antennas at $\mathrm{BS}$, it is observed that the NOMA based MIMO system 
has superior performance than OMA based MIMO systems with the proposed user set selection algorithm. Specifically, for $N=2$ and $K=200$, the proposed user set selection with NOMA achieves approximately $23 \mathrm{bps} / \mathrm{Hz}$, whereas the proposed user set selection with OMA provides approximately 16bps/Hz. Similarly, for the case $N=4$ and $K=200$, the proposed user set selection with NOMA achieves approximately $36 \mathrm{bps} / \mathrm{Hz}$, while the proposed user set selection with OMA attains approximately $28 \mathrm{bps} / \mathrm{Hz}$. Furthermore, the performance results show that increasing number of BS antennas improves the sum data rate for both NOMA and OMA based MIMO systems.

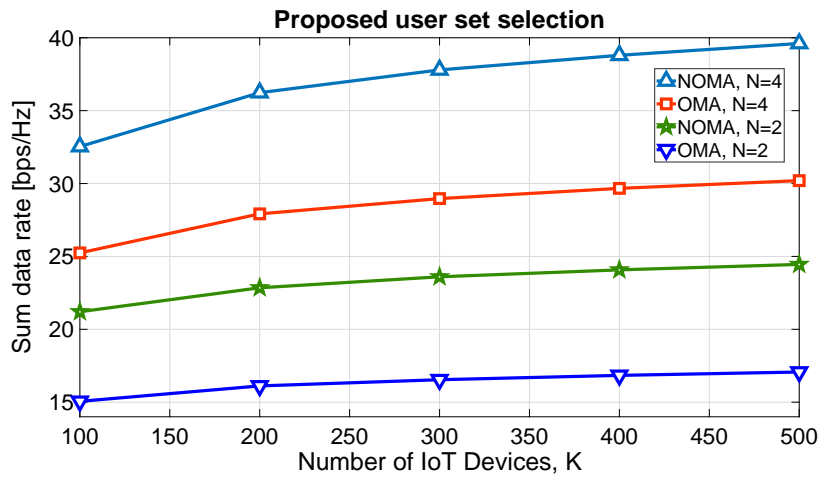

Fig. 5: The sum data rate of NOMA and OMA for $N=2$ with $\beta=0.7$ and $N=4$ with $\beta=0.6$.

For different number of BS antennas, the proposed user set selection algorithm is compared to the sorting algorithm after determining the optimal power allocation factors as presented in Section III.B. The sorting algorithm determines the strong and weak user sets by considering only their channel gains. Firstly, $K$ IoT devices are sorted based on the channel gains in descending order and classified into two groups as in (17) and (18). Using (17), the first $N$ IoT devices which have the highest channel gains are assigned to the strong user set, while using (18), the first $N$ IoT devices which have the highest channel gains are assigned to the weak user set.

As given in Fig. 6, the proposed user set selection algorithm for $N=2$ with $\beta=0.7$ provides a better performance, in terms of the data rate of the strong users, than the sorting algorithm. The reason is that the proposed user set selection algorithm considers the orthogonality between the weak user set and the strong user set, which reduces inter-set interference for the strong users. Specifically, the proposed user set selection algorithm achieves higher data rate considering the strong users around $1.3 \mathrm{bps} / \mathrm{Hz}$ compared to the sorting algorithm. Besides, the upper bound is provided assuming there is no inter-set interference for NOMA based MIMO systems in Fig. 6.

In Fig. 7, the performance of the proposed NOMA based MIMO-PNC is shown based on cumulative distribution function (CDF) with respect to the target BER. The results are provided for $K=4$ and $K=8$ IoT devices which transmit their data simultaneously to the BS equipped with $N=2$ and $N=4$ antennas, respectively. Considering target BER requirements of $6 \mathrm{G}$ systems, the applicability of the PNC-

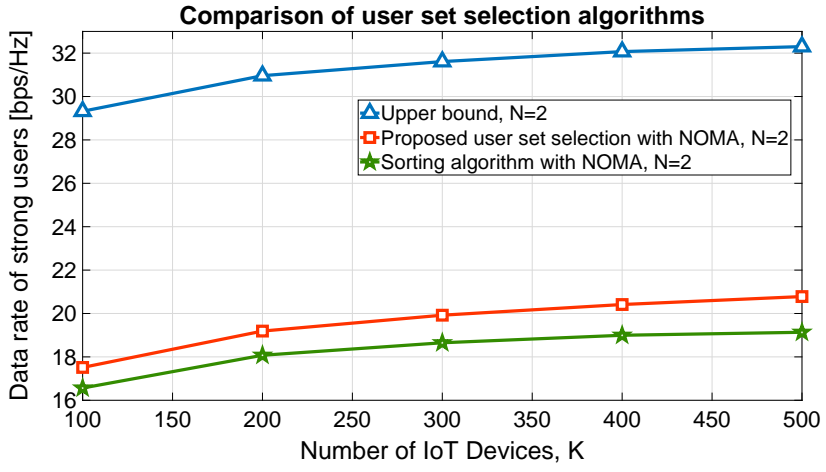

Fig. 6: The data rate of strong users versus the number of IoT devices for $N=2$ with $\beta=0.7$.

MIMO in NOMA systems is demonstrated efficiently in highly deployed IoT scenarios.

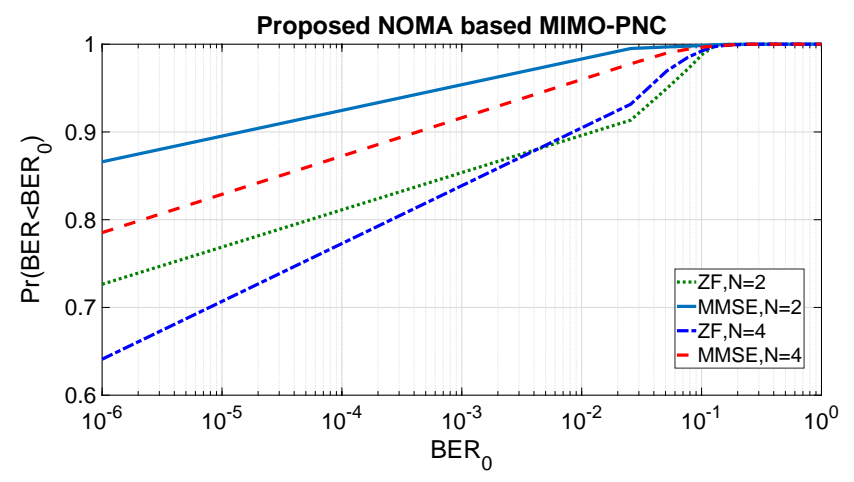

Fig. 7: CDF of BER for $N=2$ and $N=4$.

\section{CONCLUSION}

In this paper, the uplink NOMA based MIMO system with PNC was proposed for IoT applications to improve the system performance. We examined a new user set selection algorithm which determines the IoT devices incurring the least inter-set interference. Additionally, we calculated the optimal power allocation scheme to achieve maximum sum data rates. For the NOMA based uplink MIMO system, simulation results verified the advantage of the proposed user set selection with power allocation over conventional OMA systems for massive IoT applications. Furthermore, the efficiency of the proposed combined PNC and NOMA based MIMO scheme through the proposed user-set selection was demonstrated based on the BER performances of IoT devices.

\section{ACKNOWLEDGMENT}

This work has been funded by the European Union Horizon 2020, RISE 2018 scheme (H2020-MSCA-RISE-2018) under the Marie Sklodowska-Curie grant agreement No. 823903 (RECENT)

\section{REFERENCES}

[1] C.W.Chen, " Internet of Video Things: Next-Generation IoT With Visual Sensors," in IEEE Internet of Things Journal, vol.7, no.8, pp. 6676-6685, Aug. 2020. 
[2] Z. Zhang et al., "6G Wireless Networks: Vision, Requirements, Architecture, and Key Technologies," in IEEE Vehicular Technology Magazine, vol. 14, no. 3, pp. 28-41, Sept. 2019.

[3] T. Ssettumba, A. H. Abd El-Malek, M. Elsabrouty and M. Abo-Zahhad, "Physical Layer Security Enhancement for Internet of Things in the Presence of Co-Channel Interference and Multiple Eavesdroppers," in IEEE Internet of Things Journal, vol. 6, no. 4, pp. 6441-6452, Aug. 2019.

[4] N. Zhang et al., "Physical-Layer Authentication for Internet of Things via WFRFT-Based Gaussian Tag Embedding," in IEEE Internet of Things Journal, vol. 7, no. 9, pp. 9001-9010, Sept. 2020.

[5] Yu, Z., Machado, P., Zahid, A., Abdulghani, A.M., Dashtipour, K., Heidari, H., Imran, M.A. and Abbasi, Q.H., "Energy and Performance Trade-Off Optimization in Heterogeneous Computing via Reinforcement Learning," in Electronics, vol.9, no.11, pp.1812-1814, 2020.

[6] Z. Shi et al., "Zero-forcing-based downlink virtual MIMO-NOMA communications in IoT networks, " in IEEE Internet of Things Journal, vol.7, no.4, pp. 2716-2737, April 2020.

[7] S. M. R. Islam, N. Avazov, O. A. Dobre and K. Kwak, "Power-Domain Non-Orthogonal Multiple Access (NOMA) in 5G Systems: Potentials and Challenges," in IEEE Communications Surveys \& Tutorials, vol. 19, no. 2, pp. 721-742, Secondquarter 2017.

[8] O. Shental, B. M. Zaidel and S. S. Shitz, 'Low-density code-domain NOMA: Better be regular," 2017 IEEE International Symposium on Information Theory (ISIT), Aachen, Germany, 2017, pp. 2628-2632.

[9] Z. Wei, L. Yang, D. W. K. Ng, J. Yuan and L. Hanzo, "On the performance gain of NOMA over OMA in uplink communication systems," in IEEE Transactions on Communications, vol.68, no.1, pp. 536-568, Jan. 2020.

[10] Q. Wu, W. Chen, D. W. K. Ng and R. Schober, "Spectral and EnergyEfficient Wireless Powered IoT Networks: NOMA or TDMA?," in IEEE Transactions on Vehicular Technology, vol. 67, no. 7, pp. 6663-6667, July 2018.

[11] X. Wang, J. Wang, L. He and J. Song, "Outage Analysis for Downlink NOMA with Statistical Channel State Information," in IEEE Wireless Communications Letters, vol. 7, no. 2, pp. 142-145, April 2018.

[12] L. Dai, B. Wang, Y. Yuan, S. Han, I. Chih-lin and Z. Wang, "Nonorthogonal multiple access for 5G: solutions, challenges, opportunities, and future research trends," in IEEE Communications Magazine, vol. 53, no. 9, pp. 74-81, September 2015.

[13] W. Yu, L. Musavian and Q. Ni, "Link-Layer Capacity of NOMA Under Statistical Delay QoS Guarantees," in IEEE Transactions on Communications, vol. 66, no. 10, pp. 4907-4922, Oct. 2018.

[14] M. Amjad and L. Musavian, "Performance Analysis of NOMA for Ultra-Reliable and Low-Latency Communications," 2018 IEEE Globecom Workshops (GC Wkshps), Abu Dhabi, United Arab Emirates, 2018, pp. $1-5$.

[15] S. Ali, E. Hossain and D. I. Kim, "Non-orthogonal multiple access (NOMA) for downlink multiuser MIMO systems: User clustering, beamforming, and power allocation," in IEEE access, vol. 5, pp. 565-577, 2017.

[16] H. Zhang, D. Zhang, W. Meng and C. Li, "User pairing algorithm with SIC in non-orthogonal multiple access system," 2016 IEEE International Conference on Communications (ICC), Kuala Lumpur, Malaysia, 2016, pp. 1-6.

[17] S. Liu, C. Zhang and G. Lyu, "User selection and power schedule for downlink non-orthogonal multiple access (NOMA) system," 2015 IEEE International Conference on Communication Workshop (ICCW), London, UK, 2015, pp. 2561-2565.

[18] Y. N. Ahmed, "A Novel Scheduling Technique for NOMA in 5G Wireless Communication Systems," 2019 12th German Microwave Conference (GeMiC), Stuttgart, Germany, 2019, pp. 59-62.
[19] Q. Liu, W. Zhang, S. Ding, H. Li and Y. Wang, "Novel Secure Group Data Exchange Protocol in Smart Home with Physical Layer Network Coding," in Sensors, vol. 20, no:4, pp.1138, Feb. 2020.

[20] P. Chen, Z. Xie, Y. Fang, Z. Chen, S. Mumtaz and J. J. P. C. Rodrigues, "Physical-Layer Network Coding: An Efficient Technique for Wireless Communications," in IEEE Network, vol. 34, no. 2, pp. 270-276, March/April 2020

[21] S. Zhang, S. C. Liew, and P. P. Lam, "Physical-layer network coding" in Proceedings of the 12th Annual International Conference on Mobile Computing and Networking, New York, NY, USA, Sep. 2006, pp. 358-365.

[22] R. H. Y. Louie, Y. Li, and B. Vucetic, "Performance analysis of physical layer network coding in two-way relay channels," in GLOBECOM IEEE Global Telecommunications Conference, Honolulu, HI, USA, Nov. 2009, pp. 1-6.

[23] M. Lu, Z. Shen, D. Guo, and X. Wang, "A new physical layer network coding de-noising mapping based on M-QAM," in First International Conference on Electronics Instrumentation and Information Systems (EIIS), Harbin,China, Jun. 2017, pp. 1-5.

[24] V. Namboodiri, K. Venugopal, and B. S. Rajan, "Physical Layer Network Coding for Two-Way Relaying with QAM," in IEEE Transactions on Wireless Communications, vol. 12, no. 10, pp. 5074-5086, Oct. 2013.

[25] K. Lu, S. Fu, Y. Qian, and H. Chen, "SER Performance Analysis for Physical Layer Network Coding over AWGN Channels," GLOBECOM 2009 - 2009 IEEE Global Telecommunications Conference, Honolulu, HI, USA, Nov. 2009, pp. 1-6.

[26] A. C. Marcum, J. V. Krogmeier, D. J. Love and A. Sprintson, "Analysis and Implementation of Asynchronous Physical Layer Network Coding," in IEEE Transactions on Wireless Communications, vol. 14, no. 12, pp. 6595-6607, Dec. 2015.

[27] S. Zhang and S. C. Liew, "Physical layer network coding with multiple antennas," in IEEE Wireless Communication and Networking Conference, Sydney, Australia, Apr. 2010, pp. 1-6.

[28] B. Okyere, L. Musavian, and R. Mumtaz, "Multi-User Massive MIMO and Physical Layer Network Coding," in IEEE Globecom Workshops (GC Wkshps), Waikoloa, HI, USA, Dec. 2019, pp. 1-6.

[29] C. Y. Ho and C. Y. Leow, "Cooperative Non-Orthogonal Multiple Access with Physical Layer Network Coding," in IEEE Access, vol. 7, pp. 44894 44902, 2019.

[30] T. Yang, L. Yang, Y. J. Guo and J. Yuan, "A Non-Orthogonal MultipleAccess Scheme Using Reliable Physical-Layer Network Coding and Cascade-Computation Decoding," in IEEE Transactions on Wireless Communications, vol. 16, no. 3, pp. 1633-1645, March 2017.

[31] B. Kim et al., "Uplink NOMA with Multi-Antenna," 2015 IEEE 81st Vehicular Technology Conference (VTC Spring), Glasgow, UK, May. 2015, pp. 1-5.

[32] S. S. Yilmaz and B. Özbek, "Compressive Sensing based Low Complexity User Selection for Massive MIMO Systems," 2020 IEEE 91st Vehicular Technology Conference (VTC2020-Spring), Antwerp, Belgium, May. 2020, pp. 1-5.

[33] A. Grace. "Optimization Toolbox: for Use with MATLAB: User's Guide,“ Math Works Inc., Nov. 1990.

[34] R. Abozariba, M. K. Naeem, M. Patwary, M. Seyedebrahimi, P. Bull and A. Aneiba, "NOMA-based Resource Allocation and Mobility Enhancement Framework for IoT in next generation Cellular Networks," in IEEE Access, vol. 7, pp. 29158-29172, 2019.

[35] Y. Dai and L. Lyu, "NOMA-Enabled CoMP Clustering and Power Control for Green Internet of Things Networks," in IEEE Access, vol 8, pp. 90109-90117, 2020. 